\title{
Asociación entre interleuquinas inflamatorias y la presencia de trastornos intraventriculares de la conducción en pacientes con serología positiva para enfermedad de Chagas y función ventricular conservada
}

\author{
Association between inflammatory interleukins and intraventricular conduction disturbances \\ in patients with positive serology for Chagas disease and preserved ventricular function
}

\begin{abstract}
MARIO BRUNO PRINCIPATO ${ }^{1, \oplus, ~ A N A L I ́ A ~ P A O L U C C I 1, \odot ~, ~ S I L V I A ~ M I R A N D A ², ~, ~ M A R I ́ A ~ G A B R I E L A ~ L O M B A R D I ~ 2, \oplus, ~ G U S T A V O ~ S O S A ~}{ }^{2, \oplus,}$

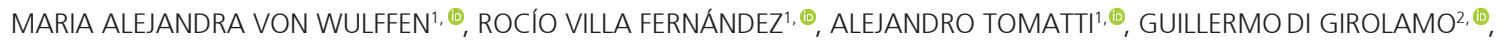
JUSTO CARBAJALES',®
\end{abstract}

\section{RESUMEN}

Introducción: Los trastornos intraventriculares de la conducción constituyen una manifestación habitual en los pacientes con enfermedad de Chagas con función ventricular izquierda conservada. Se desconoce si su presencia puede estar asociada a una mayor actividad inflamatoria.

Objetivos: Determinar si existe una correlación entre los niveles de interleuquinas y la presencia de trastornos intraventriculares de la conducción en pacientes con serología positiva para enfermedad de Chagas y fracción de eyección ventricular izquierda conservada.

Material y métodos: Se evaluó a 22 pacientes con edades comprendidas entre 21 y 80 años, seropositivos para enfermedad de Chagas, de más de 20 años de evolución y fracción de eyección ventricular izquierda mayor del 50\%. Se analizó, además, un grupo control de 14 individuos sanos. Se determinaron las concentraciones en plasma de IFN- $\gamma$, IL-1 $\beta$, IL-6, IL-10, IL-12 (p70), IL-15, IL-17A, MCP-1/CCL2, MIP-1 $\alpha /$ CCL3, TNF- $\alpha$ e IL-2. Se consideró trastornos intraventriculares de la conducción a la presencia de bloqueo de rama derecha, hemibloqueo anterior izquierdo o bloqueo de rama izquierda.

Resultados: De los 22 pacientes con serología positiva para enfermedad de Chagas, 10 presentaron trastornos de la intraventriculares de la conducción $(45,4 \%)$. En el grupo con trastornos intraventriculares de la conducción, se observaron niveles elevados de interleuquinas de alto efecto inflamatorio como INF- $\gamma$, IL-15, IL-2 (p70), IL-12, MP1- $\alpha$, en comparación al grupo control, además de presentar altos valores de IL-10 como mecanismo modulador de una respuesta inmunitaria excesiva. Conclusiones: La asociación entre niveles elevados de interleuquinas y la presencia de trastornos intraventriculares de la conducción plantea un posible proceso inflamatorio crónico para su desarrollo en pacientes chagásicos con fracción de eyección ventricular izquierda conservada.

Palabras claves: Cardiomiopatía chagásica - Interleuquinas - Trastorno del sistema de conducción cardíaco

\begin{abstract}
Background: Intraventricular conduction disturbances are common in patients with Chagas disease and preserved left ventricular ejection fraction, but their association with higher inflammatory activity is unknown.

Objectives: The aim of this study was to determine the presence of an association between interleukin levels and intraventricular conduction disturbances in patients with positive serology for Chagas disease and preserved left ventricular function. Methods: Twenty-two patients between 22 and 80 years of age with positive serology test for Chagas disease with more than 20 years progression and left ventricular ejection fraction $\geq 50 \%$ were included in the study and compared with a control group of 14 healthy individuals. Plasma levels of IFN- $\gamma$, IL-1 $\beta$, IL-6, IL-10, IL-12 (p70), IL-15, IL-17A, MCP-1/CCL2, MIP-1 $\alpha /$ CCL3, TNF- $\alpha$ and IL-2 were measured in patients and controls. Right bundle branch block, left anterior hemiblock or left bundle branch block were considered intraventricular conduction disturbances.

Results: Among the 22 patients with positive serology for Chagas disease, 10 presented intraventricular conduction disturbances (45.4\%). This group had elevated levels of interleukins with high inflammatory effect such as INF- $\gamma$, IL-15, IL-2, IL-12, MIP-1 $\alpha$, compared with the control group, and high levels of IL-10 as a regulatory mechanism of an excessive immune response. Conclusions: The association between elevated levels of inflammatory interleukins and intraventricular conduction disturbances suggests that chronic inflammation may play a role in the development of these abnormalities in patients with positive serology for Chagas disease and preserved left ventricular ejection function.
\end{abstract}

Key words: Chagas Cardiomyopathy - Interleukins - Cardiac Conduction System Disease

Rev Argent CARDIol 2021;89:130-134.http://dx.doi.org/10.7775/rac.es.v89.i2.19866

VER ARTÍCULO RELACIONADO Rev Argent Cardiol 2021;89:89-91. http://dx.doi.org/10.7775/rac.es.v89.i2.20183

Recibido: 25/11/2020 - Aceptado: 19/01/2021

Dirección para separatas: Dr. Mario Principato - Hospital General de Agudos José María Ramos Mejía - Gral. Urquiza 609, C1221 ADC, Buenos Aires, Argentina - E-mail: mbprincipato@yahoo.com.ar

Fuentes de financiamiento: Agradecemos a Novartis Argentina por el Grant que permitió la realización de este estudio Este trabajo obtuvo el "Premio de la Fundación Dr. Pedro Cossio 2020"

${ }^{1}$ Hospital General de Agudos J. M. Ramos Mejía

${ }^{2}$ Instituto Alberto C. Taquini de Investigaciones en Medicina Traslacional UBA-FMED-CONICET 


\begin{tabular}{ll|ll} 
Abreviaturas & \\
BRD & Bloqueo de rama derecha & IL1 & Interleuquina-1 \\
BRI & Bloqueo de rama izquierda & IL3 & Interleuquina-3 \\
ECh & Enfermedad de Chagas & T. cruzi & Trypanosoma cruzi \\
FEVI & Fracción de eyección ventricular izquierda & TIVC & Trastornos intraventriculares de la conducción \\
HBAI & Hemibloqueo anterior izquierdo & TNF- $\alpha$ & Factor de necrosis tumoral alfa \\
IL & Interleuquina & &
\end{tabular}

\section{INTRODUCCION}

La enfermedad de Chagas (ECh), también llamada "tripanosomiasis americana", es una enfermedad potencialmente mortal causada por el parásito protozoo Trypanosoma cruzi (T. cruzi). (1)

Se calcula que en el mundo hay entre seis y siete millones de personas afectadas por ECh, mayormente en zonas endémicas de 21 países de América Latina. Se transmite a los seres humanos principalmente por las heces o la orina de insectos triatominos (vía vectorial), los cuales presentan variados nombres, según la zona geográfica donde se encuentren. (2)

Del total de los pacientes afectados con ECh, se estima que entre el $15 \%$ y el $30 \%$ desarrollan miocardiopatía chagásica. Otras manifestaciones son los trastornos de la conducción cardíaca, manifestaciones gastrointestinales y trastornos neurológicos periféricos, centrales y autonómicos. $(3,4)$

El motivo por el cual la ECh se manifiesta de manera tan disímil, responde a diversas teorías, muchas de las cuales se complementan entre sí y continúan en constante estudio. Sin embargo, conocer el modo en que evolucionará la enfermedad continúa siendo impredecible.

Una de las ramas más estudiadas es la que representa a la actividad inmunológica. Los anticuerpos de inmunoglobulina $\mathrm{G}$ circulantes (IgG) contra los receptores muscarínicos M2 (Auto AC M2) han sido asociados a la presencia de disautonomía temprana en pacientes con enfermedad chagásica crónica, con manifestaciones clínicas tales como bradicardia sinusal o anomalías en la conducción auriculoventricular. (5-7)

Otra línea de investigación la constituye el estudio de los autoanticuerpos contra receptores $\beta 1$ y 2 que pueden ser producidos por los pacientes con miocardiopatía chagásica. (8) Estos autoanticuerpos, originalmente dirigidos contra el parásito, reconocerían indistintamente antígenos similares en el huésped, un fenómeno conocido como "mimetismo". La relación entre su concentración y el grado de afectación cardiológica (arritmias complejas, disfunción ventricular, trastornos de la conducción) continúa siendo controvertida. (9)

La acción de las citoquinas y su concentración fue otro de los factores inmunológicos que ha sido evaluado, mayormente en las etapas avanzadas de la enfermedad. Sin embargo, ha sido escasamente estudiada en etapas tempranas o asintomáticas. En algunos estudios, se observó una correlación significativa entre los niveles de algunas citoquinas inflamatorias en plasma como IFN- $\gamma$, factor de necrosis tumoral alfa (TNF- $\alpha$ ) e IL-6 y la disfunción ventricular. Por el contrario, se hallaron niveles bajos de IL-10 en los pacientes con disminución de la fracción de eyección. (10)

Se desconoce si existe una relación entre el perfil de citoquinas, su concentración y la presencia de trastornos de la conducción en las fases precoces de la enfermedad.

\section{OBJETIVOS}

El objetivo de este estudio es determinar el nivel de distintas citoquinas, tanto proinflamatorias como reguladoras en pacientes con ECh crónica asintomáticos con fracción de eyección normal y evaluar su relación con los trastornos intraventriculares de la conducción (TIVC).

\section{MATERIAL Y MÉTODOS}

Se evaluaron 44 pacientes con ECh. Se incluyeron aquellos con edades comprendidas entre 21 y 80 años, seropositivos para enfermedad de Chagas, con una antigüedad de la infección de más de 20 años conocida por el paciente, certificada por el médico tratante o por análisis serológico. Además, presentaban fracción de eyección ventricular izquierda (FEVI) mayor o igual al 50\% valorada por ecocardiograma Doppler color, dentro de los 12 meses previos a su inclusión. En el caso de presentar marcapasos definitivo, una estimulación ventricular inferior al $50 \%$.

Se constituyó, además, un grupo control de 14 individuos sanos de similar edad e igual proporción de sexos, con serología para ECh negativa.

Fueron excluidos pacientes que rechazaron firmar el consentimiento informado, con patologías conocidas graves (excluida la cardiovascular) que generaran una expectativa de vida menor de un año, que estuvieran participando en protocolos de investigación 30 días antes de la toma de la muestra, aquellos con los que no se pudiera asegurar su contacto personal o telefónico. Se excluyeron, además, sujetos con abuso de alcohol o drogas en los últimos 6 meses, clínica y laboratorio de insuficiencia hepática (valor de transaminasas tres veces por encima del valor normal y bilirrubina $>2$ $\mathrm{mg} / \mathrm{dL}$ ); con FEVI menor del 50\% que recibieran drogas de conocida acción sobre los parámetros cardiovasculares considerados (inmunosupresores, nitratos, estrógenos, estatinas, antiinflamatorios no esteroideos, otros antiinflamatorios o corticosteroides); con enfermedad coronaria en etapa aguda o crónica; con indicación de revascularización, angioplastia coronaria o cirugía de revascularización miocárdica en los 6 meses anteriores. No se incluyeron pacientes con insufi- 
ciencia renal (creatinina sérica $>3 \mathrm{~g} / \mathrm{dL}$ ); con enfermedad pulmonar obstructiva grave; con miocardiopatías dilatadas de otras etiologías. Sumado a ellos se excluyeron pacientes con diabetes, hipertensión arterial o enfermedades valvulares significativas (aunque quedaron exceptuadas las secundarias a dilatación del anillo valvular mitral o tricúspideo). Fueron excluidos pacientes con marcapasos que presentaran estimulación ventricular mayor del $50 \%$ o que tuvieran patologías autoinmunes (artritis reumatoidea, lupus, esclerodermia, hepatitis B o C, entre otras).

Luego de la firma del consentimiento informado, se confeccionó una completa historia clínica y se realizaron procedimientos diagnósticos y pronósticos correspondientes. Se efectuó ECG de 12 derivaciones y valoración de FEVI mediante ecocardiograma Doppler color.

Se procedió a la extracción de sangre en ayunas, el total obtenido $(6 \mathrm{~mL})$ se colocó en tubos Vacutainer con EDTA (Becton, Dickinson, EE. UU.) y se centrifugaron inmediatamente durante $10 \mathrm{~min}$ a $1200 \mathrm{xg}$. Se añadió inmediatamente a cada plasma un cóctel de inhibidor de proteasa recién preparado (P8340, Sigma-Aldrich) (1\% V/V). Luego se enviaron los plasmas congelados al laboratorio IATIMET para tomar alícuotas y almacenar a $-20^{\circ} \mathrm{C}$ hasta su análisis.

Las concentraciones de IFN- $\gamma$ en plasma, IL-1 $\beta$, IL-6, IL10, IL-12 (p70), IL-15, IL-17A, MCP-1/CCL2, MIP-1 $\alpha /$ CCL3, TNF-estrógenos, estatinas, e IL-2 en pacientes y controles se midieron mediante un ensayo multiplex de perlas magnéticas que emplea el panel de citoquinas/quimioquinas humano HCYTOMAG-60K (Merck Millipore, MO, EE. UU.), en un equipo Magpix ${ }^{\circledast}$. Cada muestra se cargó por duplicado. Se trazaron los estándares y se determinaron las concentraciones usando el software xPONENT versión 4.2 y se expresaron en $\mathrm{pg} / \mathrm{mL}$. Todos los pacientes recibieron tratamiento estándar acorde con las guías de tratamiento nacionales e internacionales y tuvieron total libertad de retirarse del proyecto si así lo decidiesen. Se consideraron trastornos intraventriculares de la conducción (TIVC) a la presencia de bloqueo de rama derecha (BRD), hemibloqueo anterior izquierdo (HBAI) o bloqueo de rama izquierda (BRI).

\section{Análisis estadístico}

Para el análisis estadístico, se llevaron a cabo pruebas no paramétricas: para las variables categóricas se utilizó la prueba de Chi Square y Cramer V. Para la comparación entre dos variables numéricas se utilizaron las pruebas de Mann Whitney U y Kolmogorov Smirnov Z. Se corrigió el valor crítico para las pruebas de hipótesis en 0,05 .

\section{Consideraciones éticas}

La investigación se condujo respetando la Resolución del Ministerio de Salud $N^{\circ}$ 1490/07, la letra y el espíritu de las declaraciones de Nüremberg, Helsinki y sus enmiendas y la Ley 3301 del Gobierno de la Ciudad de Buenos Aires "Protección de Derechos de Sujetos en Investigaciones". También estuvo alcanzada por la legislación del secreto médico y por la Ley $\mathrm{N}^{\circ} 25.326$ de Protección de Datos Personales.

El estudio junto con el formulario de consentimiento informado y la hoja de información para el paciente (FCI) fue aprobado por la Comisión de Ética en Investigación del Hospital J. M. Ramos Mejía. En el acto de firma del consentimiento, se entregó un original al paciente.

\section{RESULTADOS}

Se evaluaron 44 pacientes, de los cuales 22 presentaron FEVI disminuida y los restantes conservada. Este último grupo fue utilizado para el análisis. Se dividó en dos grupos, con TIVC y sin estos. Las características de la población se desarrollan en la Tabla 1.

De los 22 pacientes con serología positiva para ECh, 10 presentaron TIVC $(45,4 \%)$. Del grupo sin TIVC, la media de la edad fue de 50,92 $( \pm 7,1)$ años, con una FEVI del $62 \%( \pm 7,07)$ y el $41,7 \%$ eran hombres. Mientras que en el grupo con TIVC la edad fue 61,3 $( \pm 13,36)$ años, con una FEVI del $59,60 \%( \pm 6,28)$ y el $60 \%$ eran hombres.

Se comparó la relación entre los niveles de citoquinas de los pacientes sin TIVC con el grupo control, sin encontrarse diferencias significativas (Tabla 2). Según estas observaciones el grupo sin TIVC se comporta de manera similar a la población control.

Se observaron niveles mayores de interleuquinas 10, 2, 12 p70, 15, MP1- $\alpha$, INF $\gamma$ y MP1- $\alpha$ en el grupo con TIVC; al ser comparado con el grupo sin TIVC, mostró diferencias significativas (Tabla 3).

\section{DISCUSIÓN}

Los mecanismos inmunomediados e inflamatorios subyacentes, resultado del contacto del sistema inmunitario con el parásito, junto con las alteraciones del sistema nervioso autónomo, parecerían estar implicados en la transición de la ECh de la forma asintomática hacia la forma cardíaca.

Existen dos subpoblaciones principales de linfocitos $\mathrm{T}$ según sus marcadores y su funcionalidad, la de linfocitos T CD4+, o colaboradores, y la de CD8+, o citotóxicos, ambas implicadas en el control de la infección por T. cruzi. En un modelo de infección aguda en ratones, la eliminación de los linfocitos T CD8+ incrementó la parasitemia, en tanto que, en los humanos, la disminución de los linfocitos CD4+, como ocurre en

\begin{tabular}{|lccr|} 
& $\begin{array}{c}\text { Con trastorno } \\
\text { de la conducción }\end{array}$ & $\begin{array}{c}\text { Sin trastorno } \\
\text { de la conducción }\end{array}$ & $\mathbf{p}=$ \\
\hline $\mathrm{N}$ (pacientes) & 10 & 12 & \\
\hline Edad $(\dot{X} \pm S D$, años $)$ & $61,30 \pm 13,36$ & $50,92 \pm 7,10$ & 0,107 \\
\hline Sexo $(M, \%)$ & $6(60)$ & $5(41,7)$ & 0,392 \\
\hline Fey $(\dot{X} \pm S D)$ & $59,60 \pm 6,28$ & $62,00 \pm 7,07$ & 0,539 \\
\hline
\end{tabular}

Tabla 1. Características de la población incluida en el trabajo

Fey: Fracción de eyección. 
Tabla 2. Comparación de los niveles de interleuquinas entre pacientes con ECh sin trastornos de la conducción y grupo control

\begin{tabular}{|c|c|c|c|}
\hline Interleuquinas & Controles & Sin trastorno de conducción & $\mathbf{p}=$ \\
\hline TNF- $\alpha$ (X, mín.-máx.) & $15,19(9,8-23,48)$ & $14,53(10,78-34,76)$ & 1,00 \\
\hline IL-6 (pg/mL) (X, mín.-máx.) & $1,79(0,0-7,01)$ & $0,79(0,00-3,81)$ & 0,12 \\
\hline MPC-1 pg/mL (X, mín.-máx.) & $181,57(94,20-228,34)$ & $186,94(89,00-392,81)$ & 0,57 \\
\hline IL-10 (pg/mL) (X, mín.-máx.) & $18,48(5,45-44,40)$ & $9,83(0,00-27,71)$ & 0,09 \\
\hline MP1- $\alpha$ (pg/mL) (X, mín.-máx.) & $10,61(0,0-21,50)$ & $3,35(0,00-9,20)$ & 0,06 \\
\hline IL-12 p70 (pg/mL) (X, mín.-máx.) & $14,20(5,43-28,60)$ & $9,02(2,93-15,09)$ & 0,11 \\
\hline IL-2 (pg/mL) (X, mín.-máx.) & $3,32(1,13-5,60)$ & $2,07(1,14-3,18)$ & 0,04 \\
\hline IL-1 $\beta$ (pg/mL) (X, mín.-máx.) & $2,64(0,84-4,43)$ & $1,87(0,67-2,89)$ & 0,14 \\
\hline IL-15 (pg/mL) (X, mín.-máx.) & $6,25(1,32-12,30)$ & $3,36(1,37-7,74)$ & 0,08 \\
\hline IL-17A(pg/mL) (X, mín.-máx.) & $4,64(1,82-9,58)$ & $3,69(0,83-6,87)$ & 0,09 \\
\hline INF- $\gamma(p g / m L)(X$, mín.-máx.) & $9,33(4,62-33,46)$ & $7,25(2,43-10,55)$ & 0,07 \\
\hline
\end{tabular}

TNF- $\alpha$ : Factor de necrosis tumoral $\alpha$, IL 6: Interleuquina-6, MPC-1: Proteína quimioatrayente de monocitos 1, IL-10: Interleuquina 10, MP1- $\alpha$ : Proteína inflamatoria del macrófago $\alpha$, IL-12 p70: Interleuquina 12 con su subunidad p70, IL-2: Interleuquina 2, IL-1 $\beta$ : Interleuquina 1 beta, IL-15: Interleuquina 15, IL-17A: Interleuquina 17A, INF- $\gamma$ : Interferón gamma.
Tabla 3. Comparación de los niveles de interleuquinas en pacientes con ECh con trastornos de la conducción y sin estos la infección por virus de la inmunodeficiencia humana (VIH), permite la reactivación del parásito, lo cual indica que los linfocitos $\mathrm{T}$ son cruciales en el control de la infección. (11)

Diferentes estudios han demostrado que los linfocitos T CD4+ y CD8+ a través de sus citoquinas efectoras cumplen funciones relevantes en el control de la multiplicación de los parásitos durante las fases aguda y crónica de la infección, pero paradójicamente, tendrían un papel importante, en la patogénesis de la enfermedad chagásica crónica. A su vez, los linfocitos Th1 y Th17 diferenciados a partir de linfocitos CD4, a pesar de ser importantes en el control de la infección en fase aguda, tendrían una acción deletérea durante la fase crónica. (12)

Por su parte, los linfocitos T CD8+ participan en la citotoxicidad directa o mediante la producción de citoquinas como TNF- $\alpha$. En pacientes con mayor compromiso cardíaco, se han observado altos niveles circulantes de TNF- $\alpha$. (13)

En los pacientes con miocardiopatía chagásica crónica, se evidenció miocitolisis, infiltrado celular, fibrosis intersticial y adelgazamiento de la membrana basal de los cardiomiocitos. (14) En este infiltrado celular, se observan parches con predominio de linfocitos, especialmente T CD8+ y CD4+. (15)

Por lo tanto, la inmunidad natural y adquirida contribuyen al control parasitario y, de este modo, al riesgo cardiovascular. Los mecanismos producidos por la inmunidad natural ante la presencia del agente patógeno -en este caso, el parásito-inducen reacciones inflamatorias. La respuesta inflamatoria se asocia a la expresión de numerosas citoquinas, tales como interferón $\gamma$, interferón $\alpha$, TNF- $\alpha$, la interleuquina 1 (IL1) y la interleuquina 3 (IL3). (12) 
Algunas citocinas proinflamatorias, como el TNF- $\alpha$, producido por monocitos y linfocitos, han mostrado una correlación positiva con el grado de daño cardíaco. (16)

La evidencia demuestra que los pacientes con miocardiopatía chagásica, presentan una fuerte respuesta inmune expresada por niveles más altos de IFN- $\gamma$, TNF- $\alpha$ e IL-6 en comparación con pacientes portadores del parásito sin compromiso cardiaco y pacientes controles normales. A su vez, los pacientes sin compromiso cardíaco presentan niveles más altos de IL-10. (17)

En los pacientes con ECh crónica asintomática, se demostró la presencia de niveles más elevados de producción y secreción de IL-10 por las células T reguladoras, en comparación con pacientes con miocardiopatía. La mayor expresión de IL-10 también se correlaciona con una mejor función ventricular izquierda. (16)

En cuanto a las IL-17RA e IL-17A/F, su ausencia durante la infección por T. cruzi parecería tener como resultado un aumento del parasitismo tisular y una menor respuesta de células T CD8+ específicas de parásitos. Por lo tanto, las IL-17RA e IL-17A son consideradas factores críticos para mantener la inmunidad de las células T CD8+ a T. cruzi. (18)

En nuestro trabajo no hallamos diferencias en las concentraciones de IL17 A entre los pacientes con TCIV y sin estos. Por el contrario, se encontraron concentraciones mayores de IL10 en los pacientes con FEVI conservada y TIVC en comparación a los que no los tenía. Además, se observaron mayores concentraciones de citoquinas inflamatorias como las MP1- $\alpha$, la interleuquinas $2,12,15$ y el INF- $\gamma$ en el grupo con TIVC.

\section{CONCLUSIONES}

Los resultados de nuestro estudio indicarían que en la etapa de la ECh donde se evidencian trastornos intraventriculares de la conducción con FEVI preservada (etapa B1) se encuentra un aumento de citoquinas proinflamatorias como el MIP1- $\alpha$ la IL-2, 12, IL-15 y el INF- $\gamma$, además de niveles elevados de IL-10, que actuaría como un factor regulador del proceso inflamatorio. Por otra parte, se evidenciaron niveles elevados de citoquinas inflamatorias más activas, como la IL-6 y TNF- $\alpha$, sin alcanzar niveles de significación estadística. Hasta el momento, no se conocía esta relación entre el sistema inmune y la presencia de TIVC de la conducción en pacientes chagásicos con FEVI conservada.

\section{Declaración de conflicto de intereses}

Los autores declaran que no poseen conflicto de intereses.

(Véase formulario de conflicto de intereses de los autores en la web / Material suplementario).

\section{BIBLIOGRAFÍA}

1. La enfermedad de Chagas (tripanosomiasis americana) Organización mundial de la salud https://www.who.int/es/news-room/ fact-sheets/detail/chagas-disease-(american trypanosomiasis)

2. Rodrigo Sousa G, Assis Silva Gomes J, Gomes Fares R, de Souza Damasio M, Chaves A, Silvestre Ferreira K, et al. Plasma Cytokine
Expression Is Associated with Cardiac Morbidity in Chagas Disease. PLoS One 2014;9:e87082.

3. WHO. Weekly Epidemiological Record 2015;90:33-44.

4. Giménez L, Mitelman JE. Situación epidemiológica de la enfermedad de Chagas Mazza en Iberoamérica. 2010. https:// www.intramed. net/sitios/libro_chagas.

5. Kölliker-Frers RA, Otero-Losada M, Razzitte G, Calvo M, Carbajales $\mathrm{J}$ and Capani F. Chagas cardiomyopathy: role of sustained host-parasite interaction in systemic inflammatory burden. In: Veeranoot Nissapatorn, Helieh S. Oz (Eds.) - Chagas disease - Basic investigations and challenges, September 2018. https:/doi.org/10.5772/ intechopen.77980.

6. Thiers C, Barbosa J, Pereira B, Nascimento E, Nascimento J, Medei E, Pedrosa R. Autonomic dysfunction and anti-M2 and anti- $\beta 1$ receptor antibodies in Chagas disease patients. Arq Bras Cardiol 2012; 99:732-9.

7. Marin Neto JA, Rassi Jr A. Update on Chagas Heart Disease on the first centenary of Its discovery. Rev Esp Cardiol 2009;62:1211-6. https://doi.org/10.1016/s1885-5857(09)73346-8.

8. Rosenbaum M B, Chiale P A, Schejtman D, Levin M, Elizari M V. Antibodies to Beta-Adrenergic Receptors Disclosing Agonist-Like Properties in Idiopathic Dilated Cardiomyopathy and Chagas' Heart Disease. J Cardiovasc Electrophysiol 1994;5:367-75. https://doi. org/10.1111/j.1540-8167.1994.tb01174.x.

9. Chiale P A, Rosenbaum M B, Elizari M V, Hjalmarson A, Magnusson Y, Wallukat G, et al. High Prevalence of Antibodies against Beta 1- and Beta-Adrenoceptors in Patients with Primary Electrical Cardiac Abnormalities. J Am Coll Cardiol 1995; 26:864-9. https://doi. org/10.1016/0735-1097(95)00262-2.

10. Rodrigo Sousa G. Plasma Cytokine Expression Is Associated with Cardiac Morbidity in Chagas Disease. PLoS One 2014; 9:e87082. https://doi.org/10.1371/journal.pone.0087082. eCollection 2014.

11. Teixeira de Freitas V, Teixeira de Freitas VL, Vicente da Silva SC, Sartori AM, Bezerra RC, Visone Nunes Westphalen E, et al. Real-time PCR in HIV/Trypanosoma cruzi coinfection with and without Chagas disease reactivation: association with HIV viral load and CD4 level. PLoS Negl Trop Dis 2011;5: e1277. https://doi.org/10.1371/journal.pntd.0001277 12. Padilla A, Xu D, Martin D, Tarleton D. Limited role for CD4+ T-cell help in the initial priming of Trypanosoma cruzi-specific CD8+ T cells. Infect. Immun 2007;75:231-5. https://doi.org/10.1128/ IAI.01245-06.

13. Talvani A, Rocha M, Barcelos L, Gomes Y, Ribeiro A. Teixeira M. Elevated concentrations of CCL2 and tumor necrosis factor-alpha in chagasic cardiomyopathy. Clin Infect Dis 2004;38:943-50. https:// doi.org/10.1086/381892

14. Ferrans V, Milei J, Tomita Y, Storino R. Basement membrane thickening in cardiac myocytes and capillaries in chronic Chagas' disease. Am J Cardiol 1988;61:1137-40. https://doi.org/10.1016/00029149(88)90148-8

15. Benvenuti LA, Higuchi ML, Reis MM. Upregulation of adhesion molecules and class I HLA in the myocardium of chronic chagasic cardiomyopathy and heart allograft rejection, but not in dilated cardiomyopathy. Cardiovasc Pathol 2000;9:111-7. https://doi.org/10.1016/ s1054-8807(00)00027-2

16. Vasconcelos RH, Azevedo E de AN, Diniz GT , Cavalcanti M da GA de M , Oliveira de W Jr , Morais C N L, et al. Interleukin-10 and tumour necrosis factor-alpha serum levels in chronic Chagas disease patients. Parasite Immunol 2015;37:376-9. https://doi.org/10.1111/ pim. 12183

17. Sousa GR, Gomes JA, Fares RC, Damásio MP, Chaves AT, Ferreira KS, et al. Plasma Cytokine Expression Is Associated with Cardiac Morbidity in Chagas Disease. PLoS One. 2014; 9: e87082. https:// doi.org/10.1371/journal.pone.0087082

18. Tosello Boari J, Araujo Furlan CL, Fiocca Vernengo F, Rodríguez C, Ramello MC, Amezcua Vesely MC, et al. IL-17RA-Signaling Modulates CD8+ T Cell Survival and Exhaustion During Trypanosoma cruzi Infection. Front Immunol 2018; 9:2347. https://oi.org/10.3389/ fimmu.2018.02347. eCollection 2018. 